CARTAS AL DIRECTOR

\section{En respuesta al artículo "Bloqueo más radiofrecuencia térmica de los nervios esplácnicos. A propósito de un caso"}

\section{DOI: 10.20986/resed.2019.3714/2018}

\section{Sr. Director:}

En relación con el artículo publicado en la Revista de la Sociedad Española del Dolor, volumen 25, número 5 , titulado "Bloqueo más radiofrecuencia térmica de los nervios esplácnicos. A propósito de un caso" (1), desde el grupo de radiofrecuencia de la Sociedad Española del Dolor (SED), con el fin de incrementar el conocimiento y formación de los especialistas en intervencionismo en dolor, queremos aclarar conceptos erróneos en relación con la técnica que aparecen en dicho artículo:

1. La radiofrecuencia térmica, neuroablativa o convencional, es una técnica invasiva que ocasiona una destrucción tisular cuyo volumen está influenciado por el calibre de la aguja, punta activa, tiempo, temperatura y conductancia del tejido. La estimulación sensitiva nos orienta sobre la situación cerca del nervio a tratar, descartando con la motora estar lo suficientemente lejos de la raíz motora, hecho importante en dolor crónico no oncológico; aunque tan importante como la estimulación son las dos o tres proyecciones radiológicas de seguridad. En cambio, como tratamiento paliativo neuroablativo, en dolor oncológico ciertos targets inexorablemente podrían justificar que obtuviéramos una respuesta motora.

2. La radiofrecuencia pulsada es otra modalidad y no puede ser un sustituto de la convencional. Con ella no se produce ninguna destrucción tisular objetivable porque no es neuroablativa. Utiliza campos eléctricos grandes con temperaturas por debajo de $42^{\circ}$. Es por ello que, hipotéticamente, la radiofrecuencia pulsada se considera una técnica neuromoduladora (2).

3. Los autores no explican si estando frente a una pancreatitis crónica se ha realizado un bloqueo diagnóstico y pronóstico antes de hacer una técnica neuroablativa, por otro lado mandatoria en dolor crónico no oncológico [3].

4. No estamos de acuerdo con las conclusiones que quieren exponerse con esta técnica.

El abordaje de los nervios esplácnicos es técnicamente más difícil que el bloqueo del plexo celiaco y presenta una alta incidencia de neumotórax. Aun así, hay discrepancias entre los distintos autores acerca de la gravedad de las complicaciones de uno y otro procedimiento.

La indicación fundamental para la radiofrecuencia de los nervios esplácnicos es el tratamiento analgésico de los síndromes de dolor visceral del abdomen superior de origen no oncológico. Con la neuroablación térmica, obviamos la difusión anómala de los agentes neurolíticos más indicados como tratamiento paliativo en procesos oncológicos, y muy especialmente en cáncer de páncreas. Hay algunos autores que recomiendan realizar la radiofrecuencia térmica de los nervios esplácnicos como una buena alternativa ante el fracaso, ya sea por distorsión anatómica tumoral de la neuroablacion química del plexo celiaco en los procesos oncológicos $(4,5)$.

5. Por último, como medida de seguridad en los procedimientos sobre los nervios esplácnicos, se recomienda tener tubo de drenaje torácico en la sala de bloqueos y la solicitud de una radiografía de tórax antes del alta del paciente.

M. L. Franco Gay

FIPP- ERFSED. Grupo de radiofrecuencia de la Sociedad Española del Dolor

Correspondencia: M. ${ }^{a}$ Luisa Franco Gay

luisafrancogay@yahoo.es

\section{BIBLIOGRAFÍA}

1. Lopez Palanca S, Montalban Moreno E, Gonzalez lopez T, Mateo Cerdan C, Cuartero del pozo AB, Arcas Molina M. Bloqueo más radiofrecuencia térmica de los nervios esplácnicos. A propósito de un caso. Rev Soc Esp Dolor 2018;25(5):3034. DOI: 10.20986/resed.2016.3509/2016.

2. Cosman Er Jr, Dolensky JR, Hoffman RA. Factors that affect radiofrequency heat lesion size. Pain Medicine 2014;15(12):2020-36. DOI: 10.1111/pme.12566.

3. Puylaert M, Kapural L, Van Zundert J, et al. Pain in Chronic pancreatitis. In: Evidence-Based Interventional Pain Medicine. Oxford: Ed Van Zundert Wiley-Blackwell; 2012.

4. Waldman S. Splacnic Nerve Block: Classic two-Needle Tecnique. In: Waldman. Interventional Pain Management. $4{ }^{\circ}$ ed. Phyladelphia: Elsevier;2015. p. 360.

5. Erdine S, Talv GK. Sympathetic Blocks of the torax. In: Raj P. Interventional Pain Management. Image Guided Procedures. 2. ${ }^{\circ}$ ed. Philadelphia: Saunders Elsevier; 2008. 